PAWEŁ PĘDZICH

Warsaw University of Technology

Department of Cartography

ppedzich@gik.pw.edu.pl

\title{
Image of the World on polyhedral maps and globes
}

Abstract. Application of polyhedrons as image surface in cartographic projections has a tradition of more than 200 years. The first maps relying on polyhedrons appeared in the 19th century. One of the first maps which based on an original polyhedral projection using a regular octahedron was constructed by the Californian architect Bernard Cahill in 1909. Other well known polyhedral projections and maps included Buckminster Fuller's projection and map into icosahedron from 1954 and S. Waterman's projection into truncated octahedron from 1996, which resulted in the "butterfly" map. Polyhedrons as image surface have the advantage of allowing a continuous image of continents of the Earth with low projection distortion. Such maps can be used for many purposes, such as presentation of tectonic plates or geographic discoveries.

The article presents most well known polyhedral maps, describes cartographic projections applied in their preparation, as well as contemporary examples of polyhedral maps. The method of preparation of a polyhedral map and a virtual polyhedral globe is also presented.

Keywords: polyhedral maps, virtual polyhedral globes, cartographic projections, polyhedrons

\section{Polyhedrons in cartography}

Polyhedrons have been used in various disciplines of science, technology and art for centuries. Scientists and artists have been passionate about them. Widely known and much appreciated works of Albrecht Dürer (1471-1528) and Leonardo da Vinci (1452-1519) present drawings of polyhedrons in various contexts and different symbolism (figs. 1, 2). These works fascinates and give room for interpretations until today.

In cartography, polyhedrons are used for many various elaborations connected with modeling, spatial analyses and spatial data visualization. In cartographic projections mainly convex polyhedrons are used, among others regular polyhedrons (so called Platonic), semi-regular polyhedrons (so called Archimedean) and Catalans polyhedrons, which are dual for them.

Plato (427-347 BCE) was the first to describe five regular polyhedrons (fig. 3 ). In the Timaeus dialogue (350 BCE) he discussed how from

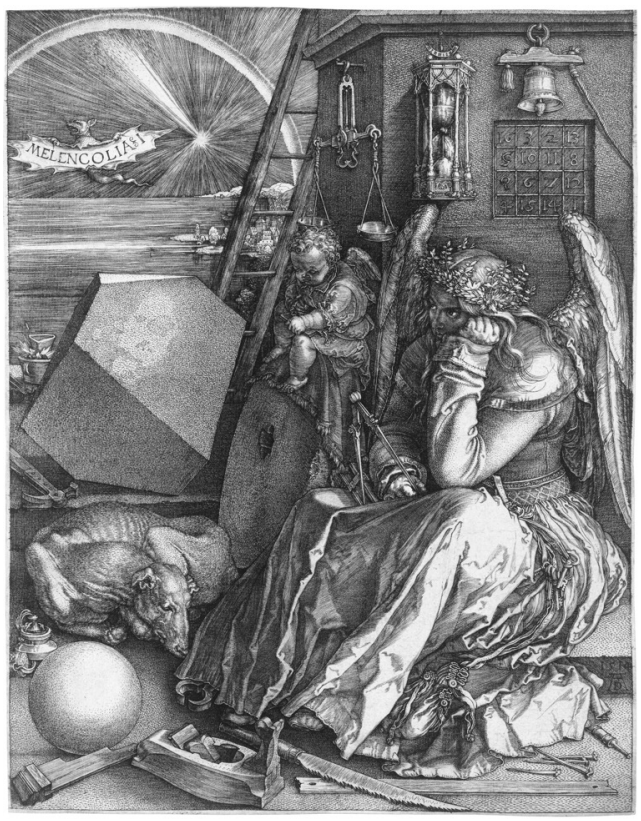

Fig. 1. Albrecht Dürer's Melancholy (source: Wikimedia) 
two kinds of triangles four polyhedrons can be created (A. Heinz 2008). He assigned them to four elements: cube to the Earth (as the least

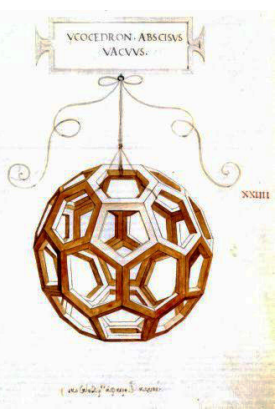

Fig. 2. Leonardo Da Vinci Ycocedron Abscisus (source: www.georgehart.com/virtual-polyhedra/ leonardo.html)

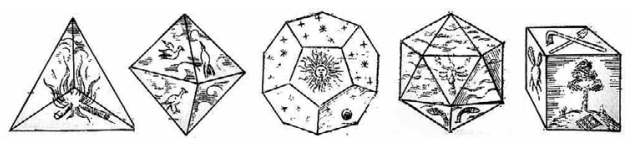

Fig. 3. Platonic solids (J. Kepler, Harmonices Mundi, 1619)

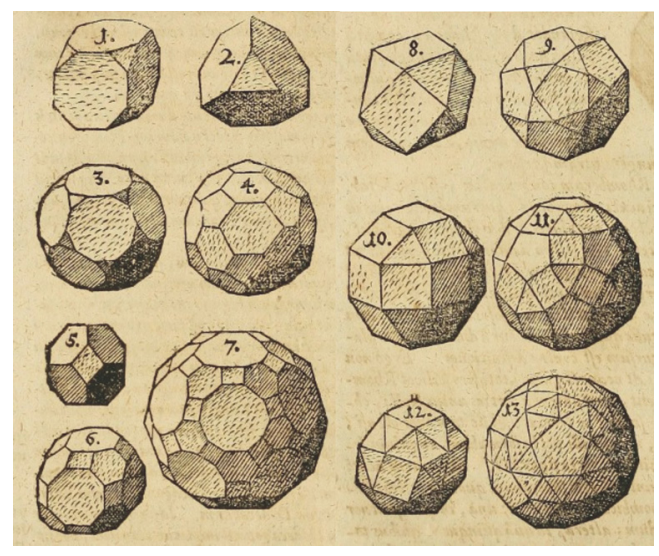

Fig. 4. Archimedean polyhedrons (J. Kepler, Harmonices Mundi, 1619)

movable, or most difficult to move), tetrahedron to fire (as the most "agile", having "sharpest cut edges and sharpest tongues in each direction"), octahedron to air and icosahedrons to water
(F. Copleston 1998). When his student Teaitet (approx. 140-368 BCE) discovered a fifth regular solid - dodecahedron, Plato immediately assumed that it was the symbol of the universe, and that God must have created it in such a shape (M. Szurek 2000). Greek names of Platonic polyhedrons: tetraedr, hexaedr, octaedr and icosaedr, as well as dodecaedr, have been used until today in mathematic terminology in almost all languages of the World except Polish (M. Szurek 2000).

The Greek mathematician Archimedes (approx. 287-212 BCE) created polyhedrons which were later called Archimedean. His works on polyhedrons have not survived until today, we can only read about them in later works of other authors. In contemporary descriptions Archimedean solids are symmetrical, semi-formed, semi-regular convex polyhedrons, i.e. their faces are regular polygons and their vertices are identical. Seven out of thirteen Archimedean polyhedrons were obtained from Platonic solids by cutting them. This meant cutting vertices and replacing them with new faces. Thirteen Archimedean solids can be distinguished (fig. 4): truncated tetrahedron, cuboctahedron, truncated cube, truncated octahedron, small rhombicuboctahedron, great rhombicuboctahedron, snub cuboctahedron, icosidodecahedron, truncated dodecahedron, truncated icosahedron, small rhombicosidodecahedron, great rhombicosidodecahedron and snub icosidodecahedron (J. D'Andrea 2011).

On each semi-regular polyhedron a sphere can be circumscribed and on each of its vertices a plane tangent to the sphere can be drawn. These planes form a convex polyhedron which is dual to the primary one. All polyhedrons dual to semi-regular polyhedrons are referred to as Catalan's polyhedrons (T. Doroziński, Z. Pogoda 2009). Figure 5 presents E. Catalan's sketches of his polyhedrons.

Another type of polyhedrons known in cartography are Waterman's polyhedrons (fig. 6), a family of polyhedrons discovered around 1990 by the mathematician Steve Waterman. Such polyhedron is created through the application of the sphere packing method within a cube. Tightly packed spheres which are further from the center than the defined diameter are removed and a convex hull is formed from the resulting set of points. This is how a Waterman's polyhedron is created. 
Jarke van Wijk (2008) introduced into cartography polyhedrons with „countless” number of faces (fig. 7). Myriahedron is a polyhedron cartographers refer to his Unterweisung der Messung and consider him a pioneer of presenting a sphere on surface using polyhedrons.

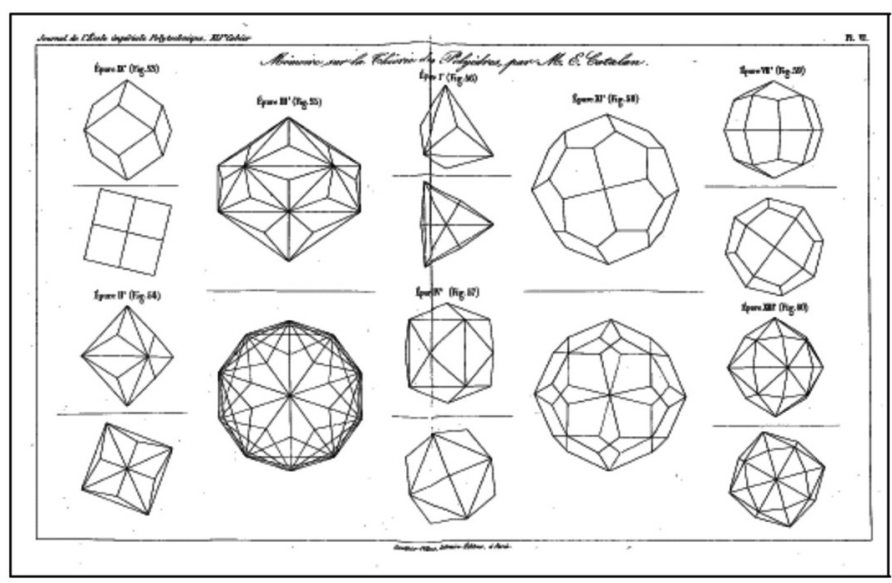

Fig. 5. Sketches of E. Catalana presenting semi-regular polyhedrons (source: M.E. Catalan 1865)
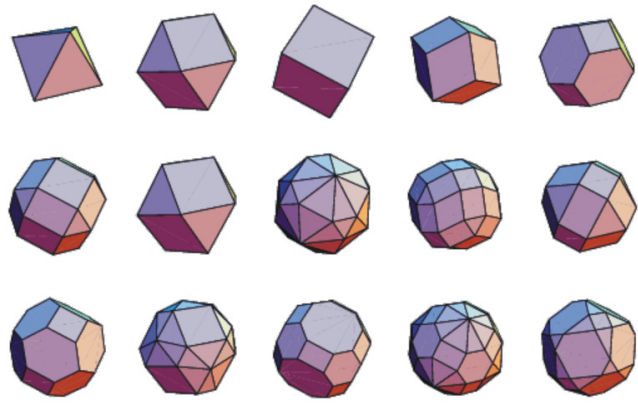

Fig. 6. Waterman's polyhedrons (source: mathworld.wolfram.com/WatermanPolyhedron.html)

with a "myriad" sides. The Latin word myriad comes from the Greek murioi meaning "ten thousand" or "countless".

Polyhedral nets form through cutting some edges and presenting them on a surface. A single polyhedron can have several nets, e.g. a cube has eleven (fig. 8). It is therefore possible to create many different maps basing on a single projection of a sphere onto a cube. One of the first works connected with presentation of polyhedral nets was published by Albrecht Dürer in Nuremberg in 1525 (fig. 9). Until today many

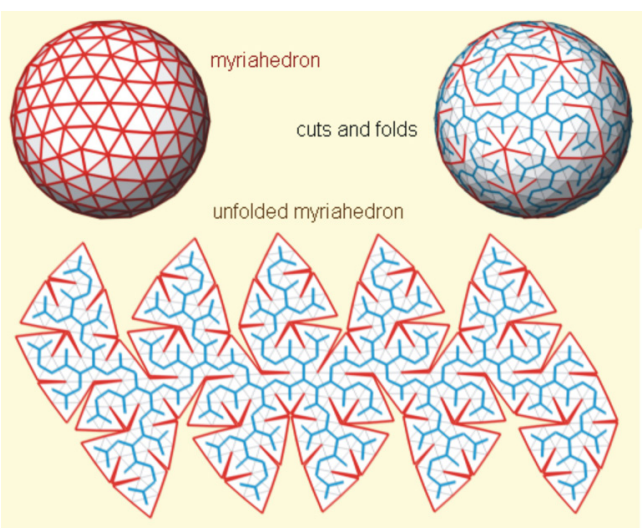

Fig. 7. Myriahedron and its net according to van Wijk (http://www.win.tue.nl/ vanwijk/myriahedral/)

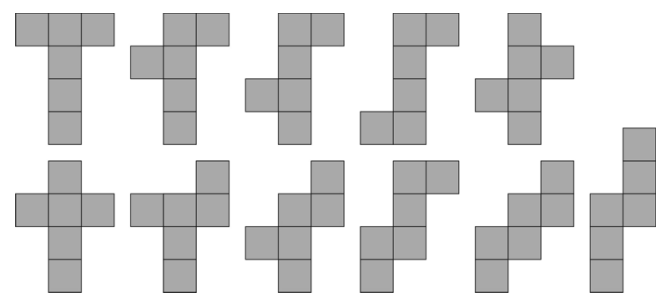

Fig. 8. Eleven nets of a cube 


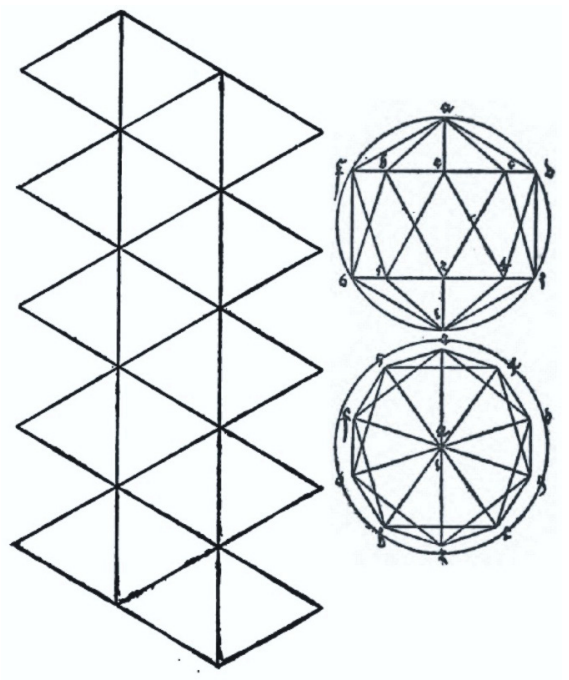

Fig. 9. Sketches of Albrecht Dürer presenting construction of a net of a regular icosahedron (source: Wikimedia)

\section{Famous polyhedral maps}

The idea of dividing a sphere into parts and projecting them separately, dates back to Leonardo da Vinci. Around 1514 the famous Italian artist, together with his collaborators, created a map of the World Sphaera Mundi in which he used curvilinear equilateral triangular octants of sphere (fig. 10). The surface of the globe was divided into eight parts forming eight spherical triangles built on the equator and two meridians differing by $90^{\circ}$. Each side of the triangle is a circular arc with its center in the opposite vertex, and each octagon is combined in groups of four around each pole. The map has no graticule. Such graticule was added to Sphaera Mundi (Paris 1551) by Oronce Fine (1494-1555), in the form of circular arcs in each octant, symmetrical to the straight line of the central meridian. The projection was also used by Guillaume Le Testu (1509-1573) to elaborate maps in 1556 and by Daniel Angelocrater (1569-1635) in 1616 (J. Snyder 1993).

Application of a polyhedron for construction of cartographic projections became an area of interest for the Californian architect Bernard J.S. Cahill (1866-1944). In 1909 he presented, and patented in 1913, a map of the World with octants arranged in such a way, that they formed a shape of a butterfly (fig. 11). Spherical lobes were limited with meridians starting from the longitude of $22^{\circ} 30^{\prime}$ west with each $90^{\circ}$ interval to minimize discontinuity of continents. B.J.S. Cahill used his own projection in which the images of meridians and equator limiting the lobes were a combination of straight lines, arcs and curves, not completely filling the equilateral triangles of the octahedron. He also published a variant of his map completely filling those triangles in conformal and gnomonic projections (fig. 12). In the case of the version basing on conformal projection he adapted a solution proposed by Oscar Adams. The author was deeply convinced that his map was superior for meteorological applications.

The "butterfly" map used to be very popular. During the Panama-Pacific exhibition in San Francisco in 1915 a competition was organized with the prize being a 90-day flight around the World. The route of that trip was presented on

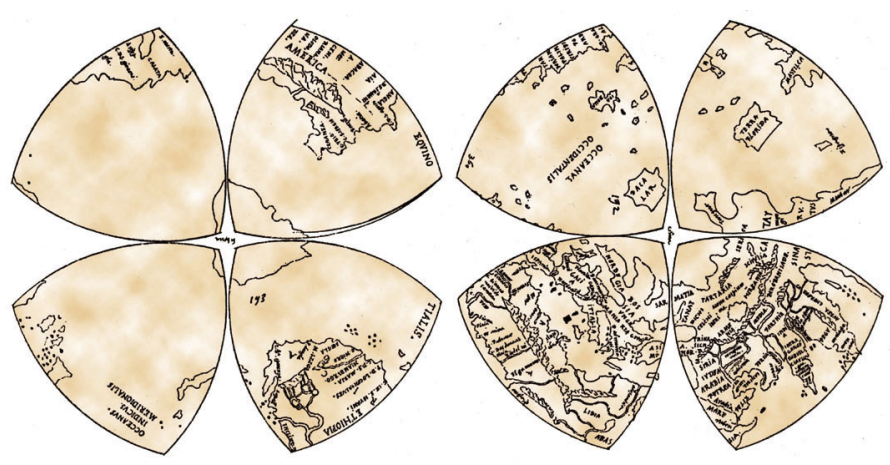

Fig. 10. Da Vinci's Map of the World (source: www.odt.org/Pictures/mappamun.jpg) 


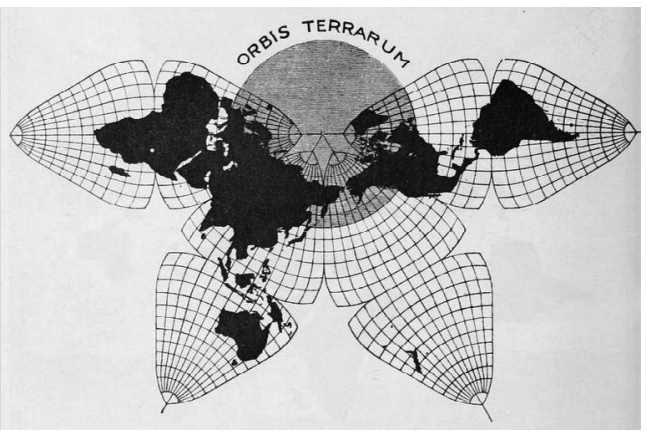

Fig 11. B.J.S. Cahill's map (source: Gene Keyes website)

a map drawn by J.S. Cahill (fig. 13). This map was awarded a gold medal at the San Francisco exhibition. Sometime later it was also used by the state of California and the city of Charleston to present ship routes (fig. 14).

In 1996 Steve Waterman, basing on Cahill's map, first published his own "butterfly" map (fig. 15). He used a truncated octahedron as the image surface and applied a sphere packing

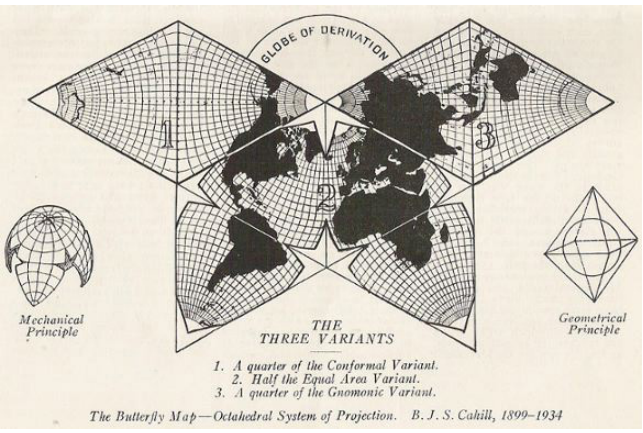

Fig. 12. Three projection types used for the construction of Cahill's map (1 - conformal, 2 - equal area, 3 - gnomonic) (source: Gene Keyes website)

own specific polyhedron and the central meridian to minimize discontinuity of the image of main continental landmasses. In the case of this version of the "butterfly" projection the meridian with the longitude of $20^{\circ}$ East was taken as the basis for dividing the sphere into octants.

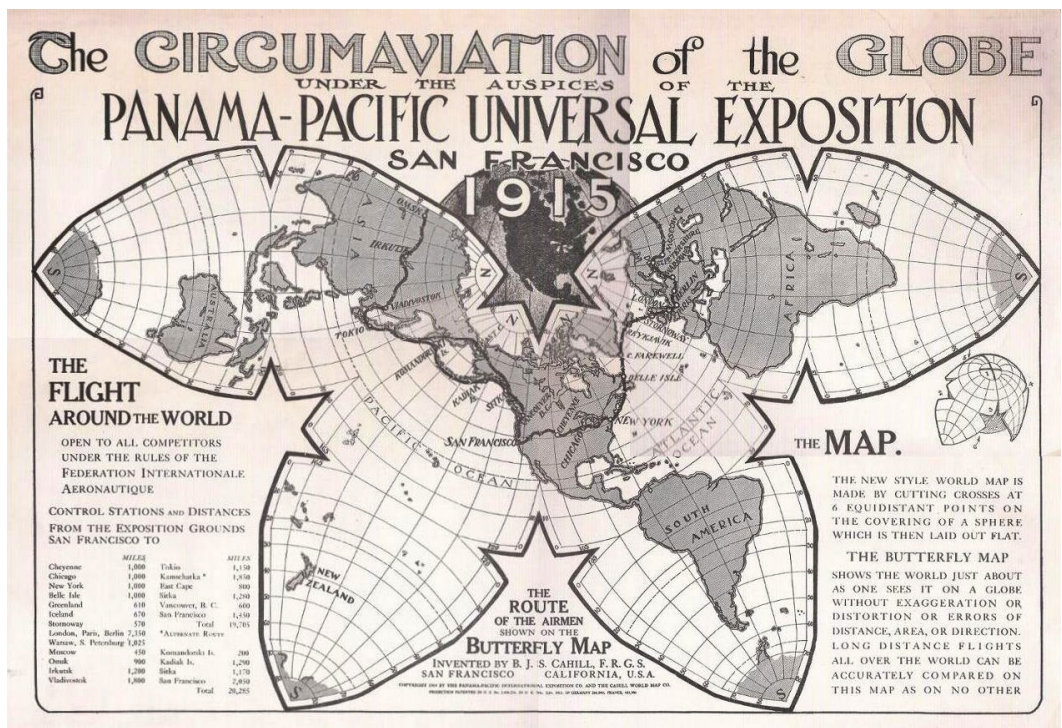

Fig. 13. Cahill's map awarded in San Francisco in 1915 (source: portal Gene Keyes website)

method which relies on connecting the centers of spheres tightly packed in a cube in order to form a convex hull. S. Waterman selected his
A map of airline routes from Dubai (fig. 16) is an example of contemporary application of a "butterfly" map. With a diagram it shows air 


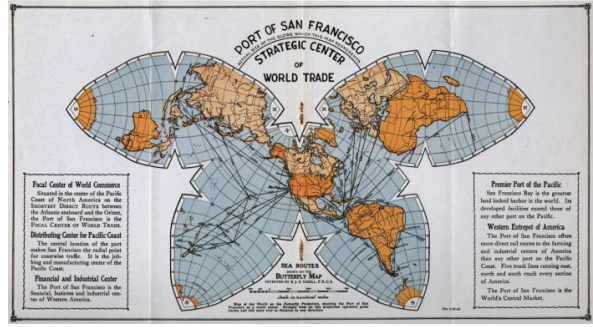

Fig. 14. Ship routes from San Francisco (source: GEO awesomeness website)

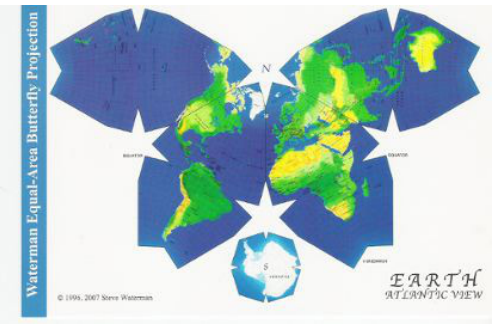

Fig. 15. S. Waterman's "butterfly" map (source: Gene Keyes Wwebsite)

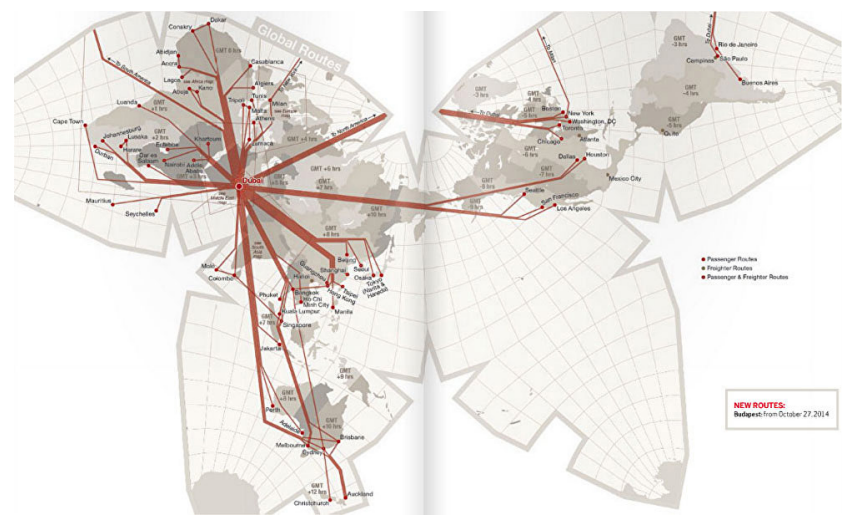

Fig. 16. Presentation of airline router from Dubai (source: D. Flynn 2014)

routes to major cities of the World, stressing that $1 / 3$ of the world's population lives within 4 hours flight to Dubai, and the remaining $2 / 3$ within 8 hours (D. Flynn 2014).

Another example of a contemporary application of Waterman's map is an animated map

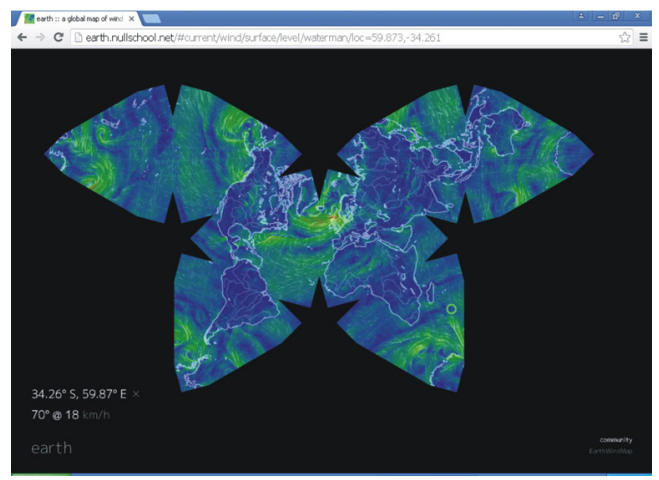

Fig. 17. Weather map in Waterman's projection (source: Nullschol.net website) presenting weather conditions in the world as well as a forecast updated every 3 hours (fig. 17). Its author is Cameron Beccario (Nullschol.net).

In 2011 the "The New York Times" published an interesting series of maps prepared in Waterman's projection presenting the fast changing world of computerization, communication and technology (fig. 18). Various aspects of this topic were presented using cartograms and choropleth maps.

Other polyhedrons were also used for map preparation. In 1943 "Life" magazine published a projection of a sphere onto a cuboctahedron. This projection was elaborated by Richard Buckminster Fuller (fig. 19). Projection of the polyhedron's edge with a constant scale of distortion is an interesting aspect of this solution (D. van Leeuwen, D. Strebe 2006). This projection was described in the article titled Life presents Buckminster Fuller Dymaxion World; it also presents fragments of the Dymaxion 


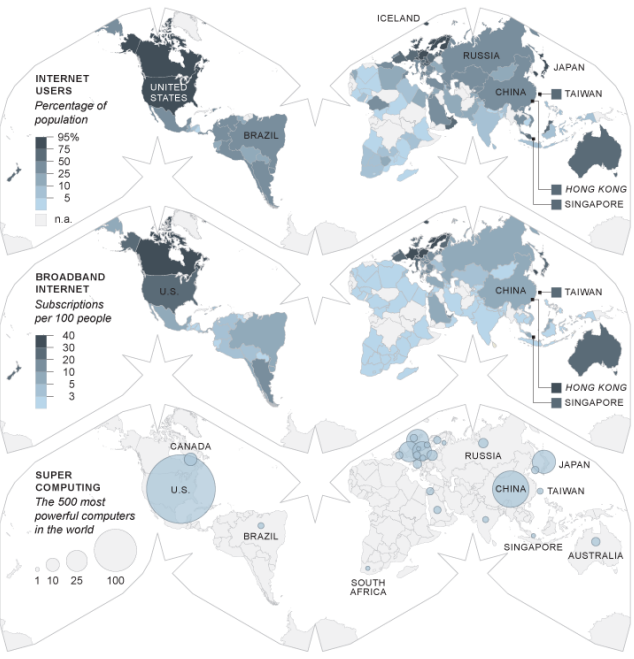

Fig. 18. "Butterfly" maps printed in the New York Times in 2011 (source: New York Times website)

map (dynamic, maximum, and tension) with instructions how to form a cuboctahedron (from eight equilateral triangles and six squares) representing the Earth's surface. In 1946 R.B. Fuller included a detailed description of his map in the patent nr US 2393676 A. It was the first two-dimensional map of the complete surface of the Earth which presented our planet as a whole, without high distortion and continents division into parts.

R.B. Fuller's Airocean World Map published in 1954, where lands are shown without breaks,

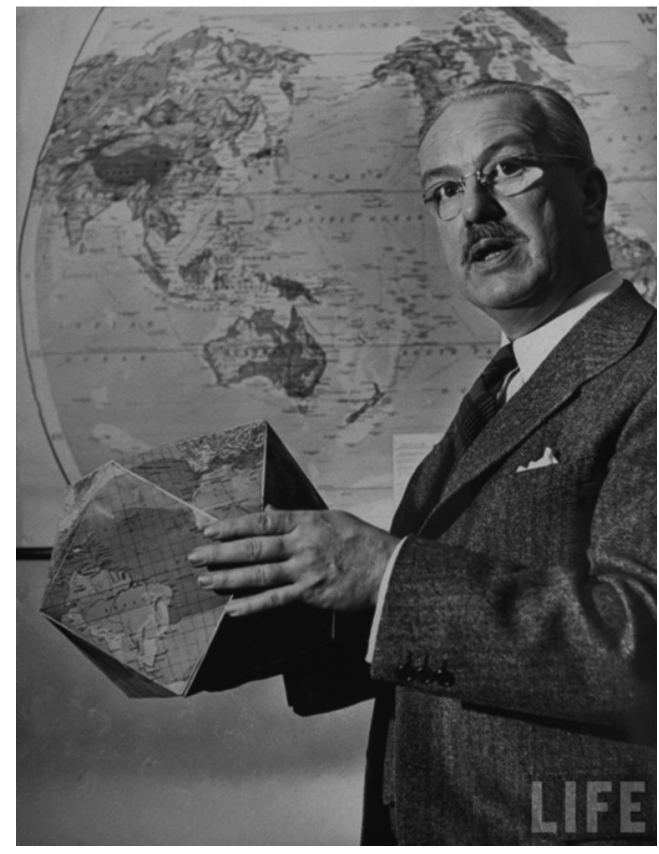

Fig. 19. R. Buckminster Fuller presenting his map on a cuboctahedron (source: R.B. Fuller 1943)

used a regular icosahedron as the basis for the projection (fig. 20). R.B. Fuller applied the same projection as before.

In 2012 in New York, on the 70th anniversary of R.B. Fuller's map publication, the Buckminster Fuller Institute announced the Dymax Redux competition to design a map which would

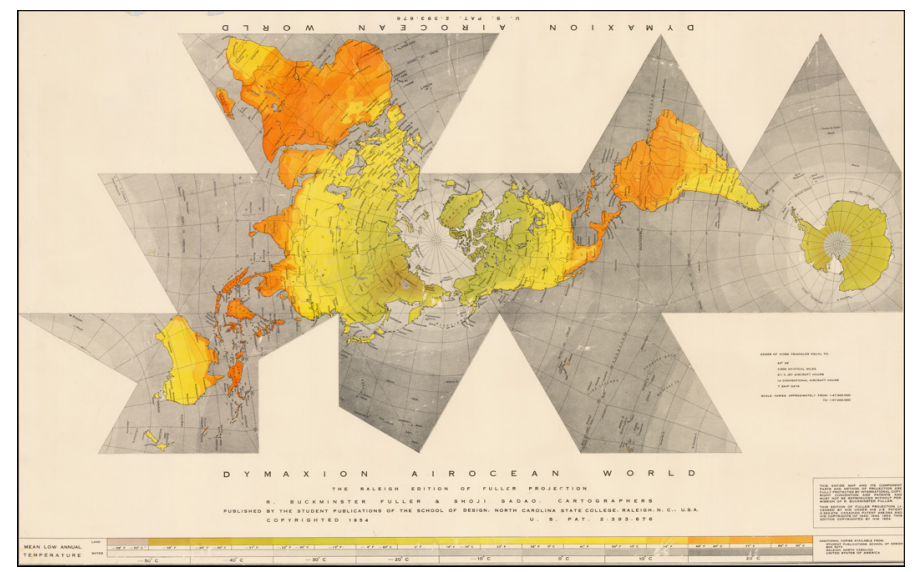

Fig. 20. Fuller's Airocean World Map (source: portal BLR Antique Maps Inc.) 
refer to R.B. Fuller's map. The winner was the team of designer Nicole Santucci and Woodcut Maps company from San Francisco. The winning project titled Dymaxion Woodocean World displays global forest densities using coloured wood textures (fig. 21). Darker shade represents higher forest density (K. Andrews 2013). and parallels (D. Van Leeuwen, D. Strebe 2006).

In his modified Lambert's azimuthal equal area projection for polyhedral maps J. Snyder (1992) modified I. Fisher's approach to discontinuity between incongruent adjacent sides. For Platonic polyhedrons, where no incongruent adjacent sides exist, J. Snyder's projection is

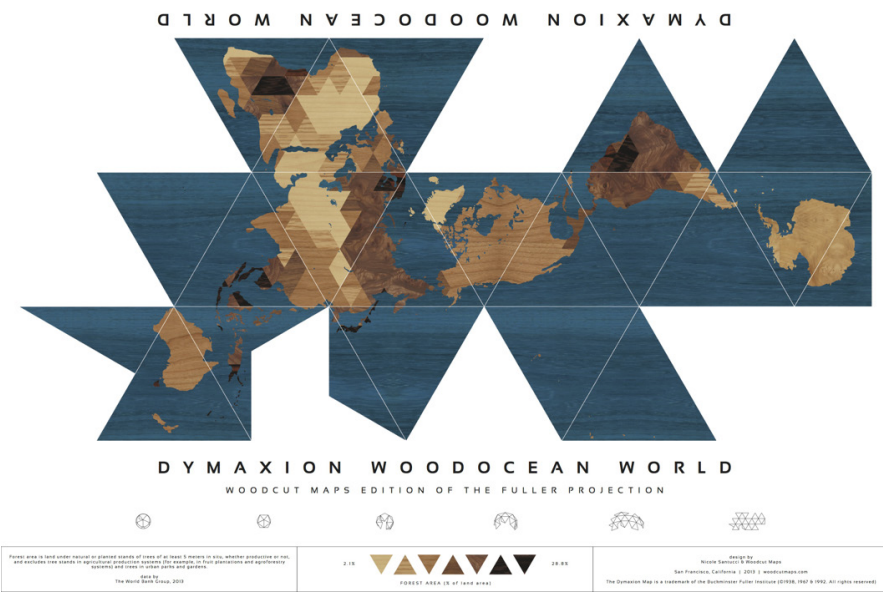

Fig. 21. Dymaxion Woodocean World map (source: Buckminster Fuller Institute website)

\section{Cartographic projection of polyhedral maps}

Most projections used to prepare polyhedral maps are gnomonic projections, but B.J.S. Cahill, R.B. Fuller and L.P. Lee used conformal projections or projections custom-designed for the purpose. L.P. Lee (1965) elaborated a conformal projection onto polyhedrons' faces using elliptic functions. This feature ensures that there are no breaks on face edges or symmetry lines. Also Amos Day Bradley (1905-1992) and Irving Fisher (1867-1947) prepared two versions of an equal area projection onto an icosahedron (J. Snyder 1993), but instead of using 20 equilateral spherical triangles Bradley divided the globe into four equal area fields which were projected onto triangles. All the projections mentioned above had breaks (discontinuity of the first derivative) along polyhedron's face edges. The unpublished projection of I. Fisher also introduces breaks alongside symmetry lines. These breaks are projected as angular points of curves - images of meridians equivalent to I. Fisher's projection. Disadvantages of J. Snyder's equal area projection include scale differences along polyhedron edges and, more significantly, serious breaks between polyhedron surfaces and along their symmetry lines.

D. van Leeuwen and D. Strebe (2006) proposed two equal area projections with certain useful features: one with constant scale on polyhedron edges and one with very small breaks along polyhedron edges. In order to obtain equal area projections a so-called sliceand-dice method was implemented.

Among other authors of this type of projections one should mention: Arthur Hinks (1821), the author of the projections onto a cube, James Smith (1939) and Stefania Gurba - onto a dodecahedron, and several more onto an icosahedrons, F.V. Botley (1949, 1954) - onto a tetrahedron and octahedron, R. Clark (1977) - onto an octahedron, A.J. Potter (1925) - onto a tetrahedron and Charles Burky (1934-1935) - onto a truncated icosahedron with 32 pentagons and hexagons (J. Snyder 1993). 
D. van Wijk is the author of myriahedral projections. Sphere is projected onto a polyhedron with numerous sides. Next the polyhedron is cut and unfolded on a plane. The resulting map has many cuts, but is almost conformal and preserves equal areas. The author presented a general approach to the selection of cut locations. He suggested three different methods: using geographical graticule, using recurrent division of the polyhedron and using geography of the Earth. The last method assumes that no continents shall be cut.

Derek H. Maling (1992) described the above polyhedral projections as polysuperficial, and the most commonly used subgroup among them as polygnomonic. Projections used for polyhedral maps consist of several image surfaces.

\section{Polyhedral projections in GIS}

Of all the popular GIS programs only ArcGIS by ESRI has built-in functions allowing for visualization of data using polyhedral projection. With this software we can prepare a map in R.B. Fuller's projection. In most GIS programs there is an option of preparing polyhedral maps using gnomonic projection. Today, when we have access to free geographic data and map editing programs, we can prepare such a polyhedral map on our own, e.g. in QGIS.

Data can be obtained e.g. from Natural Earth portal. It contains geographical data which can be used to prepare a map of the World. From the Free and Open System of Geographic Information portal one can download a free QGIS software for preparation of maps and geographical data bases.

In QGIS software there are no ready solutions for creating maps in polyhedral projections. However such maps can be elaborated by dividing the sphere of the Earth into zones by projecting the sides of a polyhedron onto a sphere. Next, one has to prepare a map in oblique gnomonic projection, separately for each zone and stick the resulting map fragments together into a whole map.

For example, we can prepare a map with its surface being a regular octahedron, i.e. similar to B.J.S. Cahill's map. A regular octahedron consists of eight equilateral triangles. The radius of the sphere inscribed into a regular octahedron equals:

$$
R=a \frac{\sqrt{6}}{6}
$$

where $a$ is the length of the side. The radius of the sphere should equal $R=6371 \mathrm{~km}$, the length of the side of the octahedron will be $a=15605,699 \mathrm{~km}$. Following B.J.S. Cahill's assumption that the division of the sphere into lobes will start at $\lambda=22^{\circ} 30^{\prime}$ meridian and then at every $90^{\circ}$, eight meridian zones are obtained (four on the northern hemisphere and four on the southern hemisphere) with axial meridians at $\lambda_{0}=22^{\circ} 30^{\prime}, 112^{\circ} 30^{\prime},-67^{\circ} 30^{\prime},-157^{\circ} 30^{\prime}$. A cartographic oblique gnomonic projection tangential at $35^{\circ} 15^{\prime} 52^{\prime \prime}$ is applied for the zones in the northern hemisphere and at $-35^{\circ} 15^{\prime} 52^{\prime \prime}$ for the zones in the southern hemisphere. Parameters of the projection of an exemplary zone declared in QGIS are as follows:

$$
\begin{aligned}
& \text { +proj=gnom +lat_0 }=35.2644444444 \\
& \text { +lon_0 }=22.5+a=6371000+b=6371000 \\
& \text { +units }=m+\text { no_defs }
\end{aligned}
$$

A map of this zone prepared in QGIS is presented in figure 22. The map as a continuous image of the whole globe is presented in figure 23. However, despite the division of the sphere into parts it shows significant distortion. Distribution of distortion in one of the zones is shown in figure 24 .

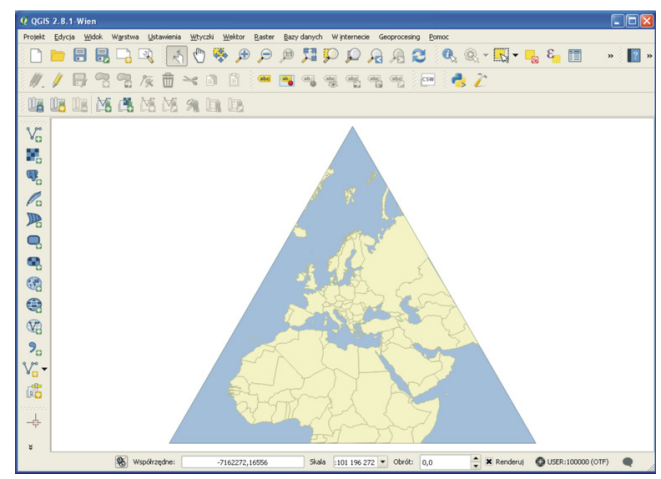

Fig. 22. One of the zones of a map in a gnomomic projection onto a regular octahedron

In the central point there is zero distortion. Highest distortion appears in triangle vertices: extreme length scales $m=1.732048$, 
$\mathrm{n}=2.999992$, area distortion scale $\mathrm{p}=5.196131$, angular distortion $\omega=31^{\circ}$.

\section{Virtual polyhedral globes}

Polyhedral globes have been known for over 200 years. In 1803 Christian Gottlieb Reichard
(1758-1837) prepared a globe in the shape of a cube, and called it Erdkubus. In 1833 J.W. Woolgar introduced a projection of the whole Earth onto a cube. In 1851 J.N. Adorno patented the globe of the Earth in the shape of an icosahedron, and in 1877 J.M. Boorman patented a series of polyhedral globes (J. Snyder 1993).

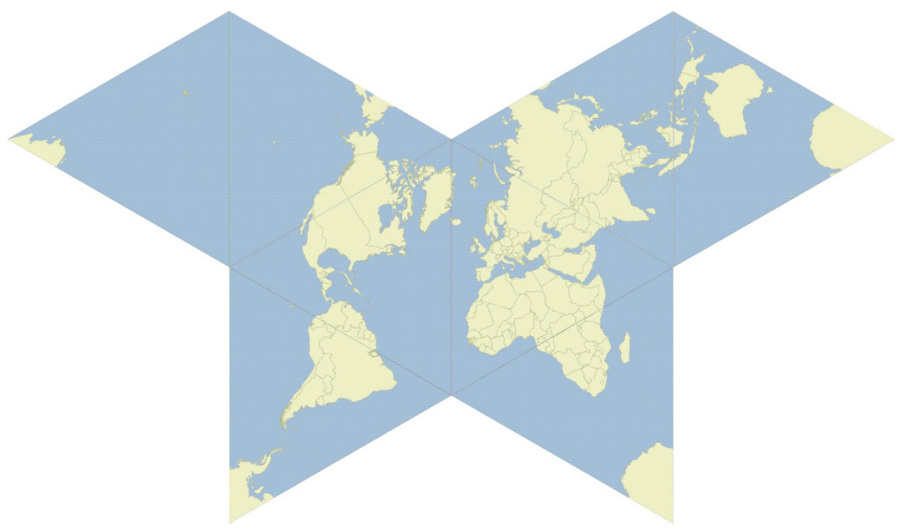

Fig. 23. Map of the World on a regular octahedron
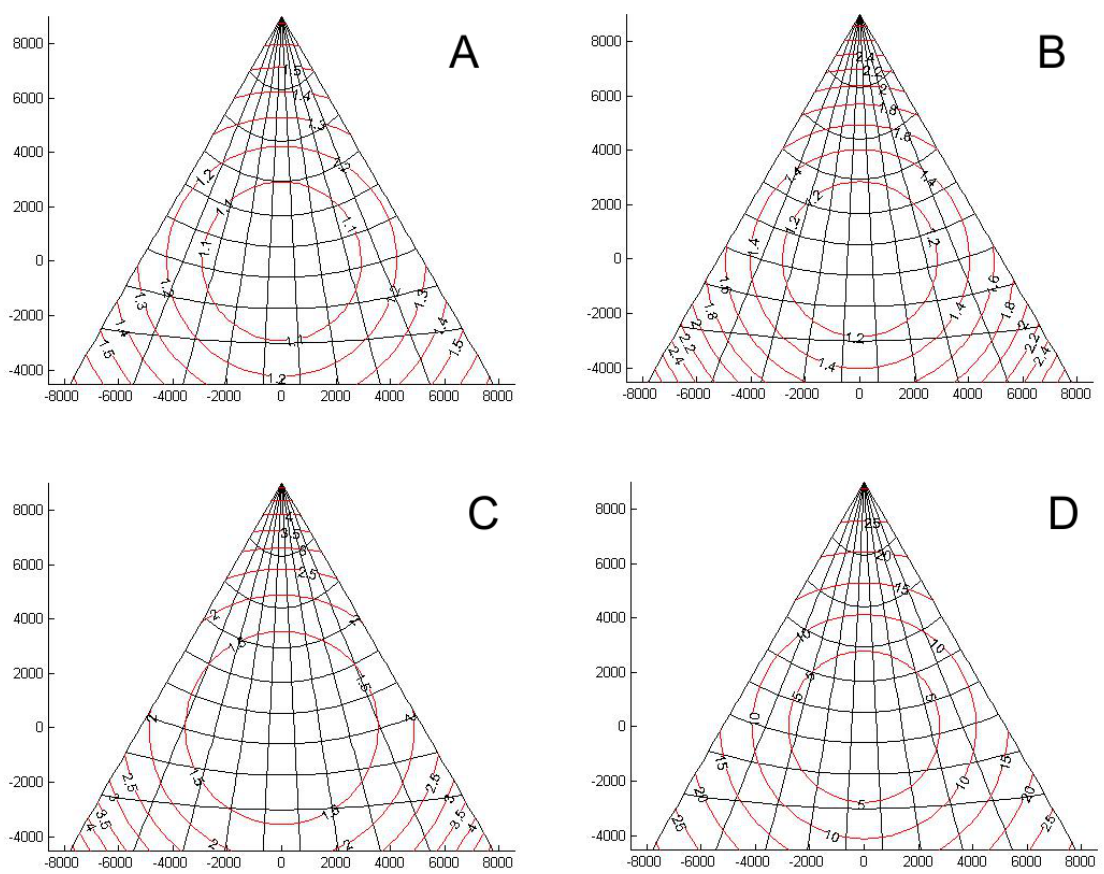

Fig. 24A. Distribution of distortion in gnomonic projection of one of the faces of a regular octahedron: A - isolines of extreme scales of distortion of length $m ; \mathrm{B}-$ isolines of extreme scales of distortion of length $n$; $\mathrm{C}$ - isolines of scales of area distortion; D - isolines of extreme angular distortion 
Polyhedral globes enjoyed popularity also in Poland. Jan Walery Jędrzejewicz (1835-1887) was the first to propose globes assembled from cardboard. In his article from 1887 he published a prototype of such a globe map (J. Jędrzejewicz 1887). In 1948 "Przełom" publishing house printed a map by Władysław Milata (1911-1954) and Wojciech Walczak (1916-1984) which could be folded into a globe. The map was divided into 20 triangles. In 1976 maps of a folding dodecahedral globe (J. Piasecka 2004) were published in the Department of Cartography at Maria Curie-Skłodowska University in Lublin. The authors were employees of the Institute: Stefania Gurba (1930-2010) and Franciszek Uhorczak (1902-1981). In a series of articles S. Gurba presented the rules of the globe preparation and its applications, such as the course of orthodrome on it (S. Gurba 1970).

At present digital maps and virtual globes are elaborated. Despite built-in options of sphere visualization many $3 \mathrm{D}$ modeling tools permit only to elaborate globes in the shape approximated with polyhedrons. For example, in VRML (Virtual Reality Modelling Language) editing a simple globe is a very easy task. It is enough to prepare a map in the Plate Carree equirectangular projection (fig. 25) and overlay it on a sphere placed in a virtual world, as a "sphere" node. However the final result may not be satis-

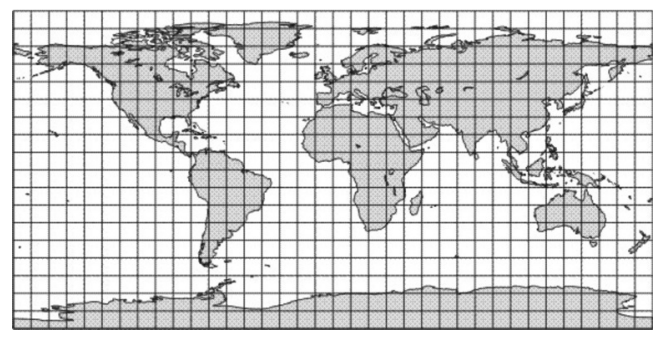

Fig. 25. Map of the World in Plate Carre projection

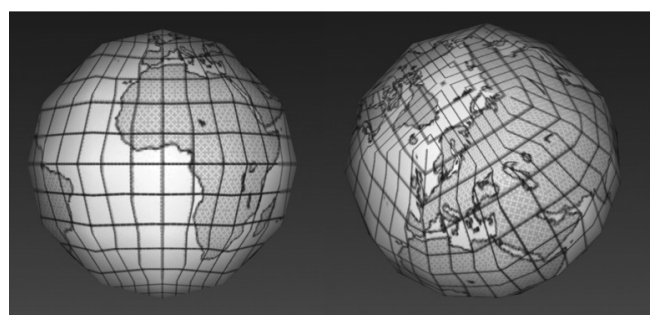

Fig. 26. Virtual globes in VRML factory (fig. 26). Numerous breaks in meridians and parallels on the globe show that the effect is far from being perfect. In order to prepare a virtual globe in this language one should rather apply one's own polyhedral construction and a map in polyhedral projection.

A 3D model of a polyhedron, e.g. a regular octahedron with a map of the World can be realized on one's own. Below is the source code in VRML and the final result in the form of a 3D model. To prepare a virtual octahedron using the below code, the map from figure 23 must be saved in JPEG format in a file named cahill.jpg.

\section{\#VRML V2.0 utf8}

Shape \{

$$
\begin{aligned}
& \text { appearance Appearance \{ } \\
& \text { texture ImageTexture }\{\text { url "cahill.jpg" }\} \\
& \text { \} } \\
& \text { geometry IndexedFaceSet \{ } \\
& \text { coord DEF COORD Coordinate }\{ \\
& \text { point }[001,010,-100,0-10,100,00-1 \\
& \text { ] } \\
& \text { \} } \\
& \text { coordlndex [0 1 2-1, } 023-1,034-1,04 \\
& 1-1,521-1,532-1,543-1,514-1 \text { ] }
\end{aligned}
$$

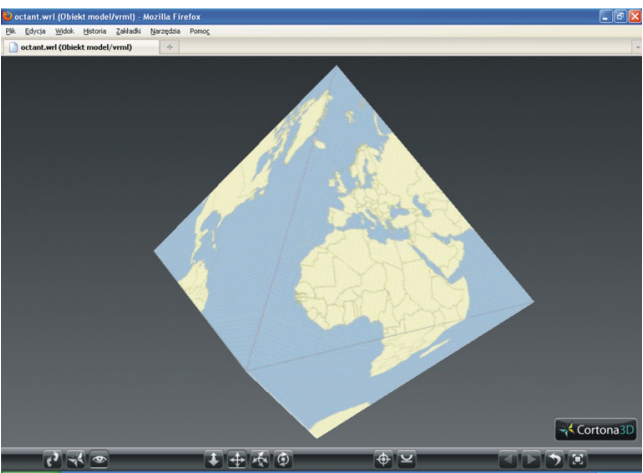

Fig. 27. Virtual regular octahedron with a map of the World 


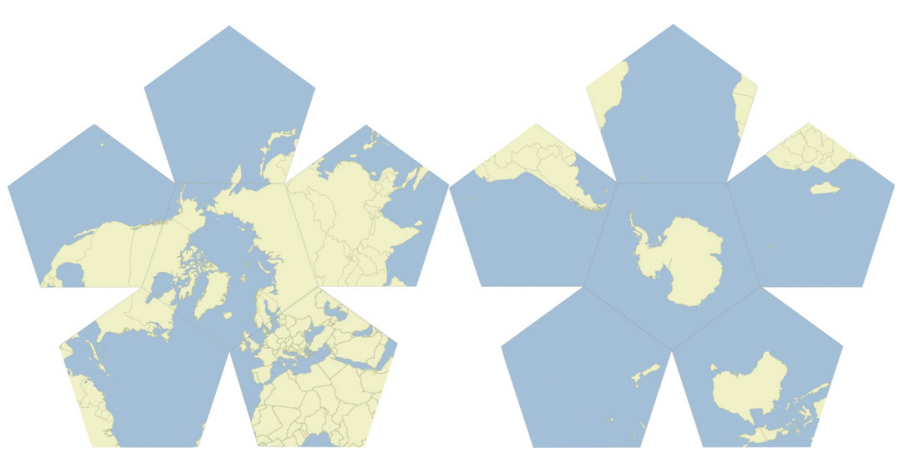

Fig. 28. Map of the World on a dodecahedron

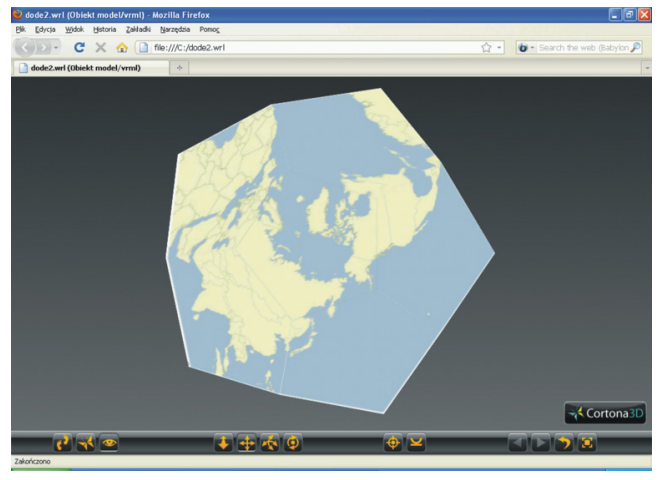

Fig. 29. Virtual dodecahedron with a map of the World

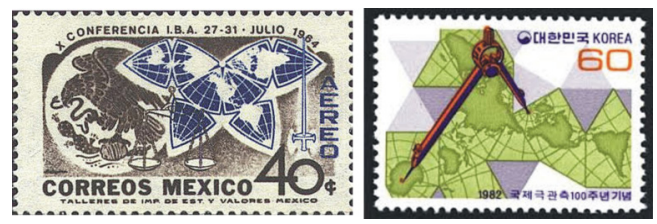

Fig. 30. Polyhedral maps on post stamps (source: portal geo-mexico)

In the above code we can distinguish two main parts. The first starts with the keywords geometry IndexedFaceSet. Here the coordinates of the polyhedron's vertices are given coord $D E F C O O R D$ Coordinate and it is defined which of polyhedron's vertices create particular triangles. In the second part, starting with texCoord DEF TEXCOORD TextureCoordinate, the coordinates of the points of division of the loaded map into triangles are given. Figure 27 presents the result of the application of the above code.

Virtual globes using different polyhedrons can be prepared analogically. Figure 28 presents a map of the World in gnomonic projection onto a dodecahedron, and figure 29 shows a virtual globe basing on it. The map was prepared following the assumptions published in the article (S. Gurba 1970). Regular and irregular polyhedrons can be formed analogically. Increasing the number of sides helps to achieve a surface approaching a sphere.

\section{Conclusions}

Polyhedrons are used in cartography to prepare maps presenting continents in continuous form and with little distortion. Maps prepared in such projections present relations between continents better than, for example, maps in the WebMercator projection used on websites. Perhaps using such maps would be a better solution than that proposed by Google. Polyhedral maps were and are very popular, especially among architects and mathematicians, who often author them. Their popularity is proven also by the fact that they appeared on post stamps (fig. 30).

Unassisted preparation of maps and globes based on polyhedrons not only helps to learn the geography of the World, but also to become familiar with various types of polyhedrons. Therefore it can find its way into the area of didactics. We can not only present image of the Earth on surface but also assemble a polyhedron from such a map. 


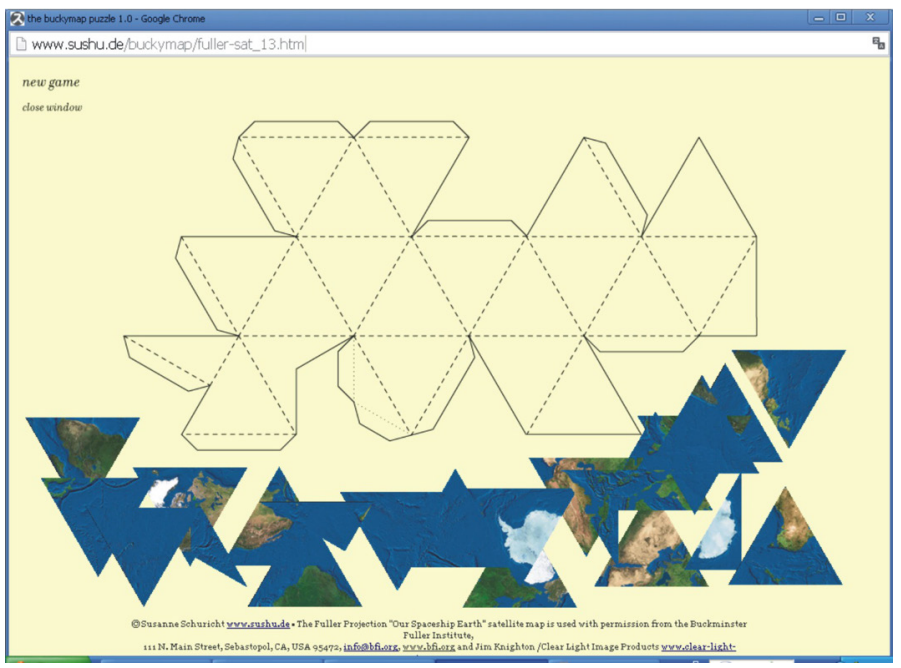

Fig. 31. Puzzle with a map of the World on a regular icosahedron (source: S. Schuricht 2001)

Globes in the form of polyhedrons are easy to construct and can be built by practically anyone, unlike spherical globes, the construction of which requires substantial knowledge and high precision. Polyhedron nets onto which the surface of the earth and other planets have been projected can be found online. For example, on Progonos.com there are ready-to-print polyhedron nets with maps of the Earth. Views of the Solar System portal which presents nets of planets and moons of the solar system is also

\section{Literature}

Andrews K., 2013, Buckminster Fuller's Dymaxion world map redesigned. http://www.dezeen.com/2013/ 08/07/buckminster-fuller-dymaxion-world-map-reimagined/ (access 10.11.2016).

Cahill B.J.S., 1909, An account of a new land map of the World. "The Scottish Geographical Magazine" no. 09, pp. 449-469.

Catalan M.E., 1865, Memoire sur la theorie des polyedres. „Journal de l'Ecole Polytechnique” vol. 24, pp. 25-71.

Copleston F., 1998, Historia filozofii. Warszawa.

D'Andrea J., 2011, Fundamental transversals on the complexes of polyhedra. A thesis for the degree of Master of Arts, Department of Mathematics, College of Arts and Sciences, University of South Florida http://scholarcommons.usf.edu/cgi/viewcontent. cgi?article $=4941 \&$ context=etd.

Doroziński T., Pogoda Z., 2009, Wielościany Catalana. „Delta” nr 12, pp. 1-3, http://mimuw.edu.pl/delta/ recommended.

Another interesting example of polyhedral maps is a puzzle on the website of Susanne Schuricht (2001), which uses a projection of the Earth surface onto an icosahedron (fig. 31).

The topic of cartographic projections is perceived as a "necessary evil" in the didactics of geodesy and cartography, but the above examples show that such issues can remain entertaining and at the same time still convey interesting information.

artykuly/delta1209/20091201-wielosciany.pdf (access 10.11.2016).

Flynn D., 2014, Emirates route map puts Dubai at the centre of the world. http://www.ausbt.com.au/ emirates-new-route-map-puts-dubai-at-the-centre-of-the-world (access 10.11.2016).

Fuller R.B., 1943, Life presents R. Buckminster Fuller's Dymaxion World. "Life" vol. 14, no. 9, pp. 41-55.

Gurba S., 1970, Ortofroma na globusie dwunastościennym. „Polski Przegląd Kartograficzny” T. 2, nr 4, pp. 160-168.

Hart G.W., 1999, Leonardo da Vinci's polyhedra. Virtual Polyhedra, http://www.georgehart.com/virtual-polyhedra/leonardo.html (access 10.11.2016).

Heinz A., 2008, Development of mathematical imagination of 3-dimensional polyhedra throughout history and inversion phenomena. 13th International Conference on Geometry and Graphics, 4-10.08.2008, Dresden. 
Jędrzejewicz J., 1887, Globus składany. „Wszechświat" T. VI , no. 27, pp. 427.

Kepler J., 1619, Harmonices Mundi. http://posner.library.cmu.edu/Posner/books/book.cgi?call=520_ K38PI (access 10.11.2016).

Maling D.H. 1992, Coordinate systems and map projections. Exeter: Pergamon Press.

Piasecka J., 2004, Polskie globusy Ziemi z XIX i XX wieku. Lublin: Wydawnictwo Uniwersytetu Marii Curie-Skłodowskiej.

Schuricht S., 2001, Exhibition of the buckymap puzzle at Buckminster Fuller - Your Private Sky exhibition, Bauhaus Dessau, Germany http://www.sushu.de/ buckymap/fuller-sat_13.htm (access 10.11.2016).

Snyder J., 1993, Flattening the earth. Two thousand years of map projections. Chicago: University of Chicago Press.

Szurek M., 2000, Matematyka dla humanistów. Warszawa: Wydawn. RTW.

van Leeuwen D., Strebe D., 2006, A "Slice-and-Dice" approach to area equivalence in polyhedral map projections. "Cartography and Geographic Information Science" Vol. 33, no. 4, pp. 269-286.

van Wijk J., 2008, Unfoolding the Earth: Myriahedral projections. "The Cartographic Journal" Vol. 45, no 1 , pp. 32-42.

\section{Internet sources (access 10.11.2016)}

BRL Antique Maps Inc, https://www.raremaps.com/ gallery/enlarge/40688

Buckminster Fuller Institute, http://bfi.org/about-bfi/ what-we-do/exhibitions/dymax-redux

Department of Mathematics, New York University http://forum.swietageometria.info/index.php?topic= 1458.0

geo-mexico.com

Gene Keynes Website :

http://www.genekeyes.com/CAHILL-1909/Cahill-1909. $\mathrm{html}$

http://www.genekeyes.com/CAHILL_GALLERY/1934Cahill-3-in-1.html

http://www.genekeyes.com/Cahill-desk-maps/3-Cahill-desk-maps.html

http://www.genekeyes.com/WATERMAN-REVIEW/ Waterman-review.html

http://geoawesomeness.com/wp-content/uploads/ 2013/12/hiddenmeanings1.jpg

http://www.math.nyu.edu/ crorres/Archimedes/Solids/solidpngs/Kepler_0073-0074.png

Natural Earth, http://www.naturalearthdata.com/ downloads/110m-physical-vectors/

New York Times, http://www.nytimes.com/interactive/2011/12/06/science/1206-world.html? r=0

Nullschol.net serwis. http://earth.nullschool.net/\#current/wind/surface/level/waterman

ODT, http://www.odt.org/Pictures/mappamun.jpg

Prognos.com, http://www.progonos.com/furuti/MapProj/Dither/ProjPoly/Foldout/foldout.html

http://www.qgis.org/pl/site/

Views of the Solar System http://solarviews.com/ eng/ico.htm

http://waaaat.welovead.com/en/top/detail/51cEgmtB.html

Wikipedia:

https://upload.wikimedia.org/wikipedia/commons/ 1/18/D\%C3\%BCrer_Melancholia_I.jpg

http://upload.wikimedia.org/wikipedia/commons/d/ db/Duerer_Underweysung_der_Messung_144.jpg 